\title{
Osteogenesis Imperfecta (Oi): A Case Report and Diagnosis Thinking Beyond Bone Fracture
}

\author{
Manuela Stoicescu* \\ Pharmacy Medical Disciplines Department, University of Oradea, Romania
}

\begin{abstract}
Objectives: Most times in the medical practice, when we have a case of bone fracture, the patient is sent to the Orthopedic Department in order to treat locally the bone fracture with bone consolidation, without thinking at other diseases, of which the patient could suffer from and which in fact represent the real cause of the fracture, exception making the women at menopause, which make frequent fractures on background of osteoporosis and the cancers with different localizations complicate with bone metastasis, in these situations the fractures appear on pathologic bone and have a reserved prognosis. We should investigate further for other pathologies in order to find the real cause of the fracture.
\end{abstract}

Methods: Present the case of a young girl aged 19, comes for a consultation with her mother with a complaint of fatigue, loss of appetite, lack of concentration and attention at school, getting tired easily after minimal physical and intellectual effort, the patient also mentions that about eight months ago she sustained a minor trauma to her left forearm which resulted in a fracture, which was then followed by a fracture of the radius and resulted in her being referred to the orthopedics department where her arm had to be fitted with metal rods. The principal signs was: bone deformation, bone shortening, thin bones, abnormally fragile bones, small muscles, joints and weak tendons, formation of thick scars, small somatic conformation, defective dentition (incomplete dentition, the teeth was damaged, the teeth falled quickly),the cornea was transparent blue(blue sclera) and also her mother had blue sclera. The molecular genetic test was used for clinic diagnosis confirmation and releaved mutations in COL1 A1 and COL1 A2 genes responsabile of sintesis of type I procollagen and confirmed the disease osteogenesis imperfecta.

Results: The case of the fracture of the forearm was a very rare genetic disease - osteogenesis imperfecta- a congenital autosomal dominance. A.D. illness and occur even if only one parent transmits the effected gene. As in this case the daughter inherited the disease from her mother.

Conclusions: Initially the case appeared to be a trivial case with a simple forearm bone fracture./Subsequently detailed physical examination revealed clinical signs such as somatic changes dentition and more importantly blue sclera pointing us towards the extremely rare disease osteogenesis imperfecta / An examination of the patient's mother revealed the same signs, confirming the patient had inherited the autosomal dominant disease from her mother./Starting from a simple fracture and some nonspecific clinical symptoms, the final diagnosis was an unexpected surprise to finally discover a hereditary disease with autosomal dominant transmission which is extremely rare - osteogenesis imperfecta. This clinical case should point out that sometimes when a simple fracture is discovered there may be previously unknown underlying disease which may have contributed to the injury and fractures can also be caused by other bone disease./ The molecular genetic test was used for clinic diagnosis confirmation and releaved mutations in COL1 A1 and COL1 A2 genes responsabile of sintesis of type I procollagen and confirmed the disease osteogenesis imperfecta.

Keywords: Bone fracture; Blue sclera; Osteogenesis imperfecta

\section{Introduction}

Most times in the medical practice, when we have a case of bone fracture, the patient is sent to the Orthopedic Department in order to treat locally the bone fracture with bone consolidation, without thinking at other diseases, of which the patient could suffer from and which in fact represent the real cause of the fracture, exception making the women at menopause, which make frequent fractures on background of osteoporosis and the cancers with different localizations complicate with bone metastasis, in these situations the fractures appear on pathologic bone and have a reserved prognosis. We should investigate further for other pathologies in order to find the real cause of the fracture.

\section{Patient and Methods}

Present the case of a young girl aged 19, comes for a consultation with her mother with a complaint of fatigue, loss of appetite, lack of concentration and attention at school, getting tired easily after minimal physical and intellectual effort, the patient also mentions that about eight months ago she sustained a minor trauma to her left forearm which resulted in a fracture, which was then followed by a fracture of the radius and resulted in her being referred to the orthopedics department where her arm had to be fitted with metal rods. The principal's signs at the objective examinations were: bone deformation, bone shortening thin bones, sometimes thick, abnormally fragile bones, small muscles, joints and weak tendons formation of thick scars. The following slides show two pictures of a postoperative scar on the forearm (Figures 1 and 2).

*Corresponding author: Manuela Stoicescu, Consultant MD, PhD, Assistant Professor, Faculty of Medicine, Pharmacy Medical Disciplines Department, University of Oradea, Romania, Tel: 0723019951; E-mail: manuela_stoicescu@yahoo.com

Received December 16, 2013; Accepted February 24, 2014; Published February 26, 2014

Citation: Stoicescu M (2014) Osteogenesis Imperfecta (Oi): A Case Report and Diagnosis Thinking Beyond Bone Fracture. J Mol Genet Med 7: 100 doi: 10.4172/1747-0862.1000100

Copyright: (C) 2014 Stoicescu M. This is an open-access article distributed under the terms of the Creative Commons Attribution License, which permits unrestricted use, distribution, and reproduction in any medium, provided the original author and source are credited 
Also on the physical examination of somatic conformation (Figure 3 ) of this girl, it is noted that she was of small stature for her age. At 19 years of age had a height of $h=1.56 \mathrm{~cm}$ and a weight of $\mathrm{G}=44 \mathrm{~kg}$, body mass index of Broca's formula BMI is much below normal for her age, leaving her with a small stature, small muscles, joints and weak tendons.

Another important signs of the objective examination were: defective dentition (Figures 4 and 5) incomplete dentition, the teeth was damaged and falled quickly. The sclera was transparent blue (Figures 6-9).

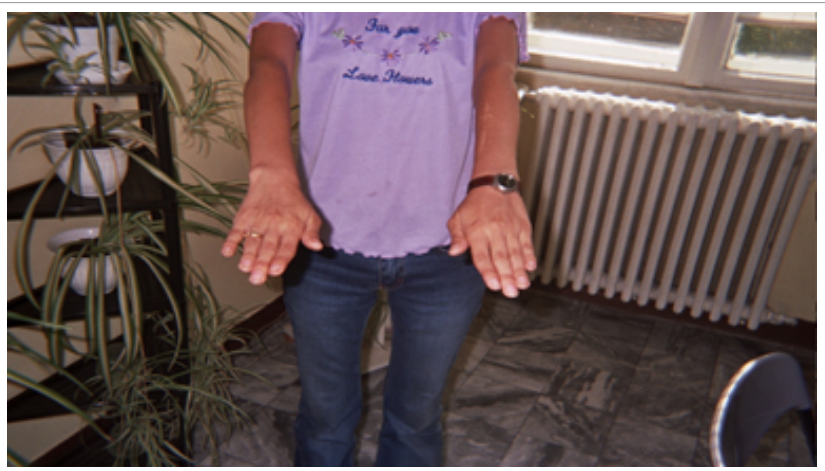

Figure 1: Scar from fracture of the left forearm.

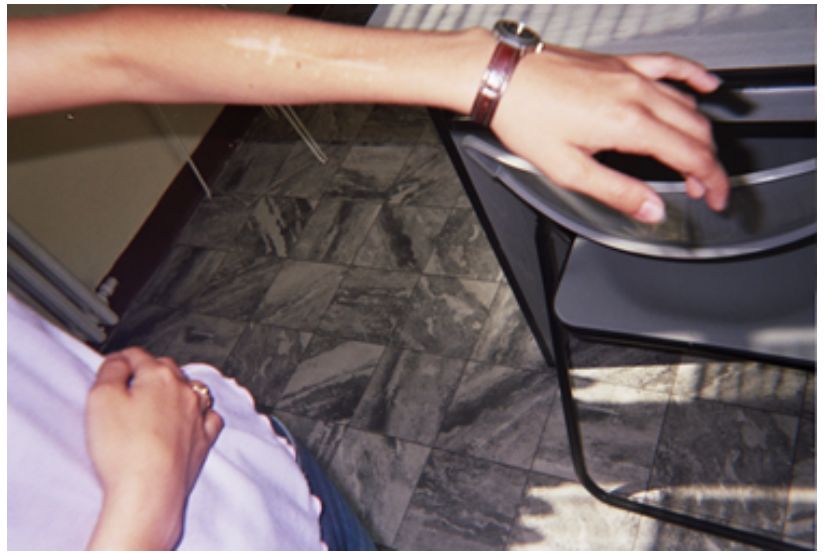

Figure 2: Post fracture scar of the left forearm.

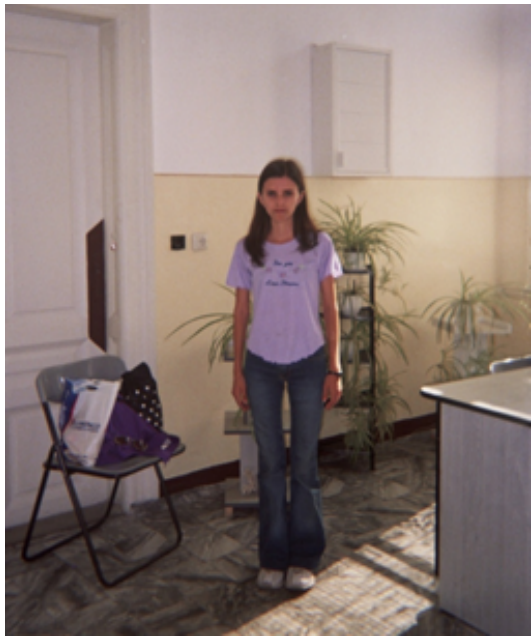

Figure 3: Somatic conformation.

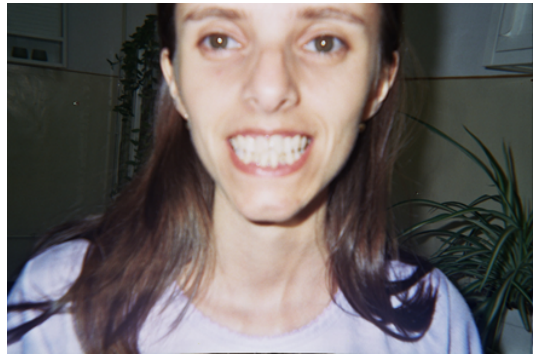

Figure 4: Teeth faulty.

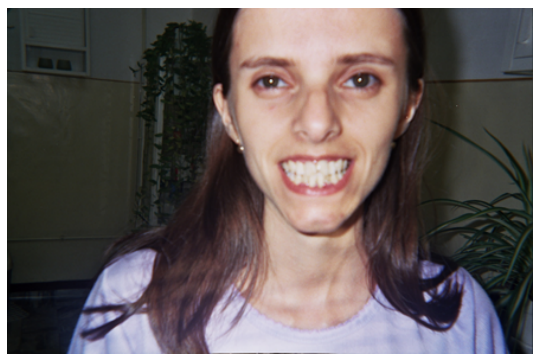

Figure 5: Changes in teeth.

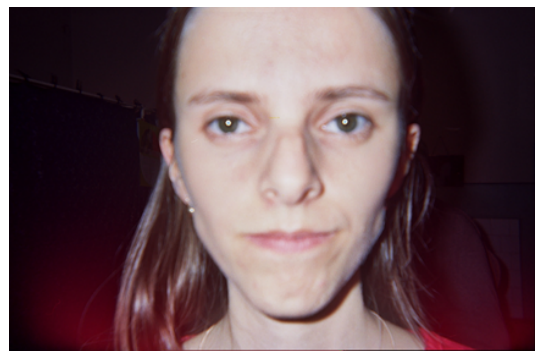

Figure 6: Blue sclera - transparent blue eyes.

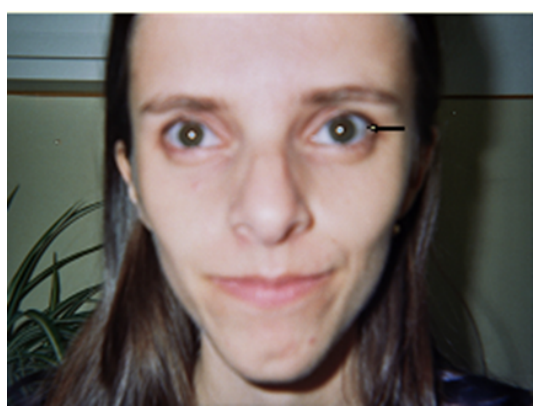

Figure 7: Blue sclera

Also her mother had blue sclera (Figures 10 and 11). The molecular genetic test was used for clinic diagnosis confirmation and releaved mutations in COL1 A1 and COL1 A2 genes responsabile of sintesis of type I procollagen and confirmed the disease osteogenesis imperfecta. The final diagnosis was osteogenesis imperfecta and fracture of the left forearm.

\section{Results}

Osteogenesis imperfecta is a congenital disorder which results 


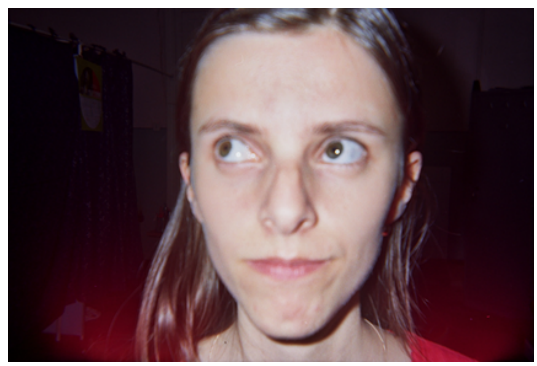

Figure 8: Blue sclera.

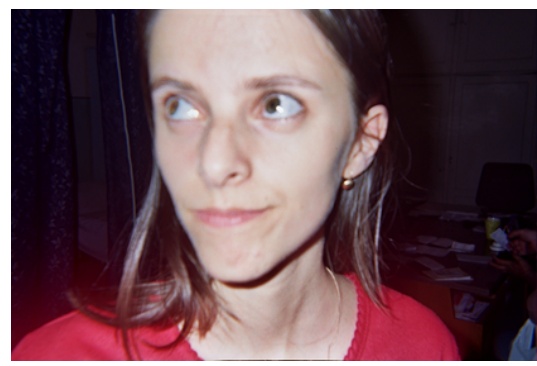

Figure 9: Blue sclera.

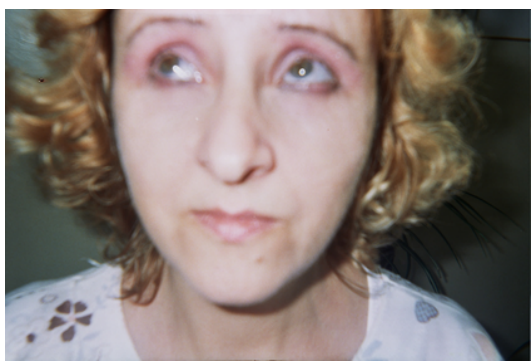

Figure 10: Blue sclera girl's mother.

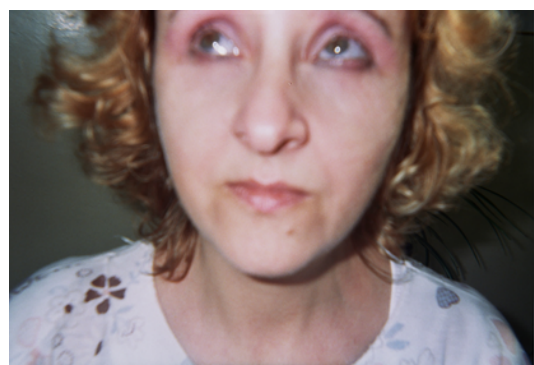

Figure 11: Blue sclera girl's mother

in the patient having extremely fragile bones. The patients will have fractures not only after they are born but these factures will occur while the baby is still in the womb. The fractures in patient will not heal easily; the child will have deformed limbs and will remain of small stature. Skull ossification is also affected in the parietal bone and neck areas; appear none ossified. If the disease occurs later in life, fractures will appear after minor traumas. Sclera will have an unusual blue color.

\section{Discussions}

Osteogenesis imperfecta is a congenital autosomal dominance.
A.D. illness can occur even if only one parent transmits the effected gene. As in this case the daughter inherited the disease form her mother. Ossification disorder is caused by metabolic disorder of collagen in connective tissue. Collagen plays an important role in bone and cartilage cell formation. Disease is present during intrauterine life, but often is diagnosed after birth.

The molecular genetic test was used for clinic diagnosis confirmation and releaved mutations in COL1 A1 and COL1 A2 genes responsabile of sintesis of type I procollagen and confirmed the disease osteogenesis imperfecta.

In these cases cultured skin fibroblasts and genetic analysis was helpful in confirming the diagnosis. Analysis of the DNA with the screening of the coding region of the COL1 A1 and the COL1 A2 genes identify the mutation. The mutations in collagen genes were change a glycine codon to that for another amino acid. The mutations appeared at $\mathrm{CpG}$ dinucleotides and are consistent with deamination of a methyl cystone to produce thymidine.

The analysis of family pedigree showed one case of OI. This genetic diseases was presented also at the grandmother of this patient and she had three fractures of the bones in she's life after minor trauma, so this genetic disease was with autosomal dominance (A.D.) transmission.

The young girl was advice to avoid pregnancy because exist risk to transmit the disease at the newborn, so genetic advice was provided.

Osteogenesis imperfecta cannot be treated with medication. However the management for this patient with OI con-sisted of supplementation with vitamin D and minerals and administration of bisphosphonates - alendronate (Fosamax) - dose for treatment was with $10 \mathrm{mg}$ once daily for six months. Fracture can be reduced with special physical therapy and careful exercise which focuses on toning muscles. If when a fracture occurs the treatment is surgical repair. Prognosis for surgical intervention is negative, because the bones are very fragile, their structure is imperfect. Bone fragility can be reduced under certain conditions by using magnesium.

If the child has osteogenesis imperfecta the child should be protected from trauma and injury. The child must learn to take care of their self and be properly informed and aware of their condition. Muscle tonification through regular exercise can have a positive effect. Bones which are most commonly affected are the long tubular bones: the femur, tibia, arm bones, radius and cubits. In this case the left radius bone was affected.

The pathogenic mechanism of this disease. There is a defect in the structure or numbers of collagen molecules. Because exist a mutation in the DNA within a gene that produce collagen this is the result of this disease osteogenesis imperfecta. The cell produce deformed collagen and developing in bad or unusual collagen fibers. The good collagen fibers, attach to bad fibers so that the rods and, consequently, the bone never becomes strong are very fragile and this is the cause of very frequent fractures of the bones after minor traumas at this category of patients.

\section{Conclusions}

1. Initially the case appeared to be a trivial case with a simple forearm bone fracture.

2. The reason this case became intriguing was the fact that the fracture occurred following such a minor trauma which suggested that there may be other underlying causes. 
3. Subsequently detailed physical examination revealed clinical signs such as somatic changes dentition and more importantly blue sclera pointing us towards the extremely rare disease osteogenesis imperfecta.

4. An examination of the patient's mother revealed the same signs, confirming the patient had inherited the autosomal dominant disease from her mother.

5. Starting from a simple fracture and some nonspecific clinical symptoms, the final diagnosis was an unexpected surprise to finally discover a hereditary disease with autosomal dominant transmission which is extremely rare - osteogenesis imperfecta.

6. This clinical case should point out that sometimes when a simple fracture is discovered there may be previously unknown underlying disease which may have contributed to the injury and fractures can also be caused by other bone disease.

7. The molecular genetic test was used for clinic diagnosis confirmation and releaved mutations in COL1 A1 and COL1 A2 genes responsabile of sintesis of tipe I procollagen and confirmed the disease osteogenesis imperfecta. 